\title{
INVESTIGATION ON AUTOMATIC CHANGE DETECTION USING PIXEL-CHANGES AND DSM-CHANGES WITH ALOS-PRISM TRIPLET IMAGES
}

\author{
A. Sasagawa ${ }^{\text {a }}$, Emmanuel Baltsavias ${ }^{\text {b, }}$, Sultan Kocaman Aksakal ${ }^{\text {b }}$, Jan Dirk Wegner ${ }^{\text {b }}$ \\ ${ }^{a}$ National Mapping Department, Geospatial Information Authority of Japan (GSI), Japan - sasagawa@gsi.go.jp \\ ${ }^{\mathrm{b}}$ Photogrammetry and Remote Sensing, ETH Zurich, Switzerland - (emmanuel.baltsavias, sultan.aksakal, \\ jan.wegner)@geod.baug.ethz.ch
}

Commission VII, WG VII/5

KEY WORDS: Change Detection, Automation, ALOS, PRISM, DSM-Change, Pixel-Change

\begin{abstract}
:
A new algorithm for automatic change detection is presented. It detects a pixel-change and DSM-change from two orthoimages and two DSMs, then it extracts the polygons in elevation-changed areas. Pixel-change is detected by using least squares fitting technique. This method can extract the visible changed areas between two orthoimages, while DSM-change is detected by difference DSM. From these two changes, polygons in elevation-changed areas are extracted using the longest matched line selection techniques. This method can automatically detect not only visible changed areas such as vegetated areas, new road construction areas and so on, but also elevation-changed areas such as new building construction, land improvement areas and so on with footprint polygon extraction. We have tested our method using the two sets of ALOS-PRISM triplet images observed over a testfield in Tsukuba, Japan. We confirmed that this method has an effect finding changed areas. Also we compared the number of extracted polygons between manual operation and our automatic method.
\end{abstract}

\section{INTRODUCTION}

Geospatial Information Authority of Japan (GSI) is the national mapping organization in Japan. Fundamental Geospatial Data (FGD) was prepared all over Japan in 2012 and now it is in the updating phase. FGD consists of 13 items such as geodetic control point, coastline, road edge, building footprint, etc. with 1:2500 scaled accuracy for city area and 1:25000 scaled accuracy for the other area.

In 1:2500 areas, FGD is updated using city plan maps, etc. which are provided by local governments. While in 1:25000 areas, there are less material data for updating. For updating FDG in 1:25000 areas effectively, it is quite important to find changed parts in widespread area at once. To solve this problem we develop the automatic change detection method using pixelchanges and DSM-changes. This time, we used the two sets of
ALOS-PRISM triplet images to confirm how our method performs well. We also give discussion on the features of our method.

\section{OUTLINE}

Figure 1 shows a flowchart of our method. Our method requires two orthoimages and two DSMs. Then it automatically provides "change detected image", which is the integrated image with pixel-change image and DSM-change image. In addition, polygons in elevation-changed area extracted from DSM-change image. Our method contains four parts (1:pixelchange extraction, 2:DSM-change extraction, 3:image integration, 4:polygon extraction in elevation changed area). Each part is described following.

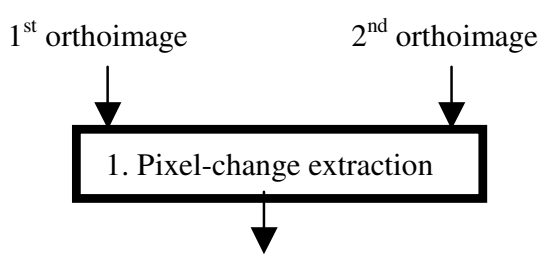

Pixel-change image

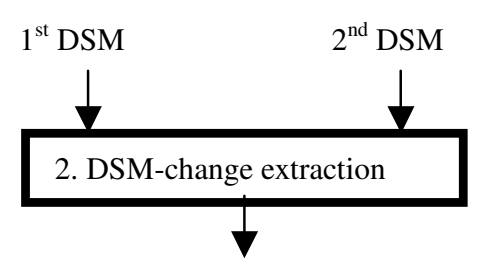

DSM-change image

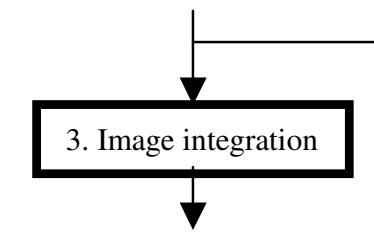

Change detected image

Figure 1:Flowchart of our change detection method

4. Polygon extraction in elevation changed area

Polygons 

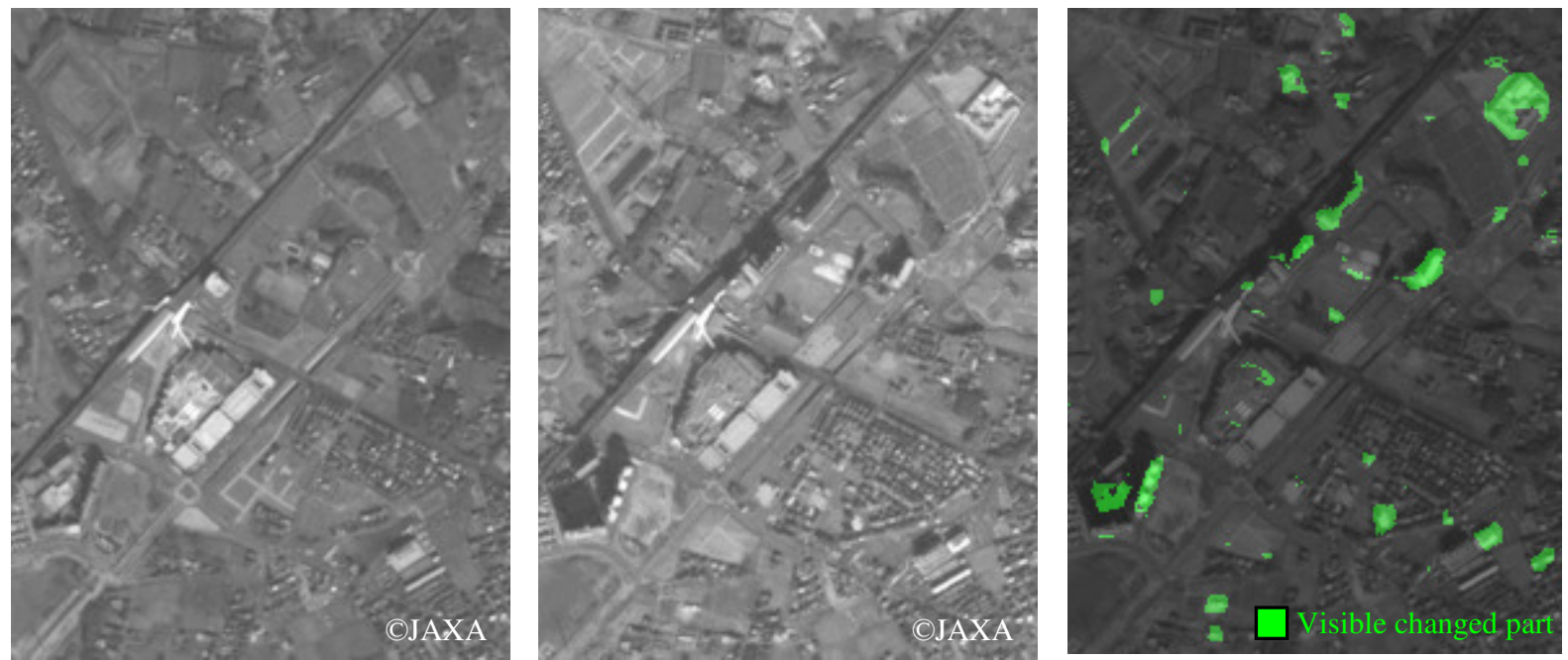

Figure 2: Example of pixel-change image

Left: $1^{\text {st }}$ orthoimage, Center: $2^{\text {nd }}$ orthoimage, Right: pixel-change image overlayed with $2^{\text {nd }}$ orthoimage

\section{OUTLINE}

\subsection{Pixel-change extraction}

In this method, Pixel-changed areas are extracted by using least square fitting technique (A.Sasagawa et al., 2008). This technique generates pixel-change index with every pixel in common area between two orthoimages. Pixel-change image shown in Figure 2 is generated from pixel-change index. Green part of the pixel-change image has large pixel change index. The size of extracted feature depends on the window size. If we use larger window it can detect large feature changes, while if we use smaller window it can detect not only small feature changes but also some small noises as shown in Figure 3.

In this case, we want to catch the changes for updating 1:25000 areas so that we don't need to extract small changes here. Due to this, we decided 25 pixels square as the window size after some trial tests.

\subsection{DSM-change extraction}

Figure 4 shows an example of 1 st orthoimage, 2 nd orthoimage and the DSM-change image. In this case, difference elevation over $+15 \mathrm{~m}$ (red part) or less $-15 \mathrm{~m}$ (blue part, but not exists in figure 4.) with over 20 square meters is extracted to catch only
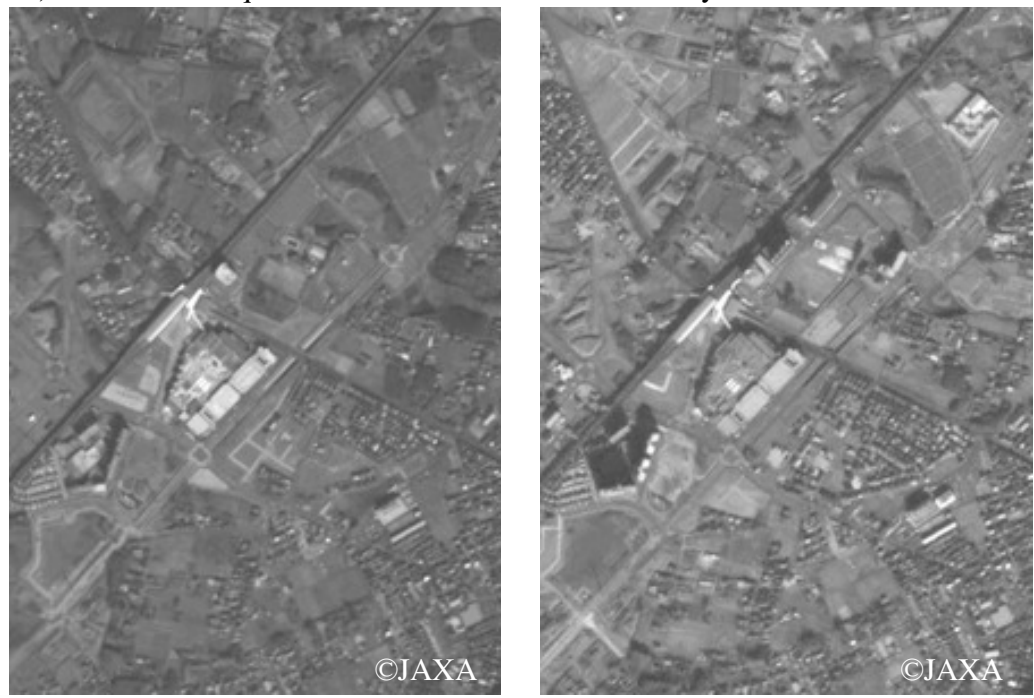
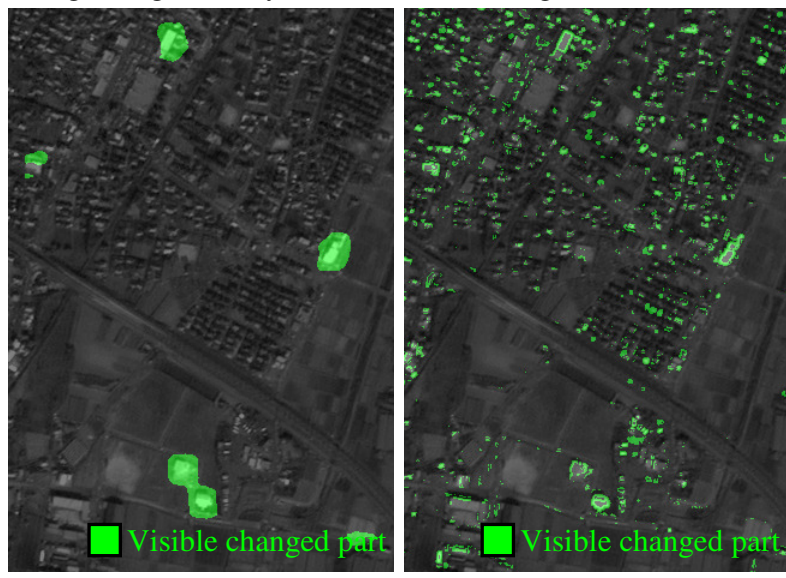

Figure 3: Pixel-change images overlayed with $2^{\text {nd }}$ orthoimage using 25 pixels square window (right) and 3 pixels square window (left)

in big changes. Each DSM is computed from each pair of nadirforward and nadir-backward overlapped image. Two DSMs were given at the same grid points. Therefore DSM-change could be realized by a calculation of the vertical difference between them.

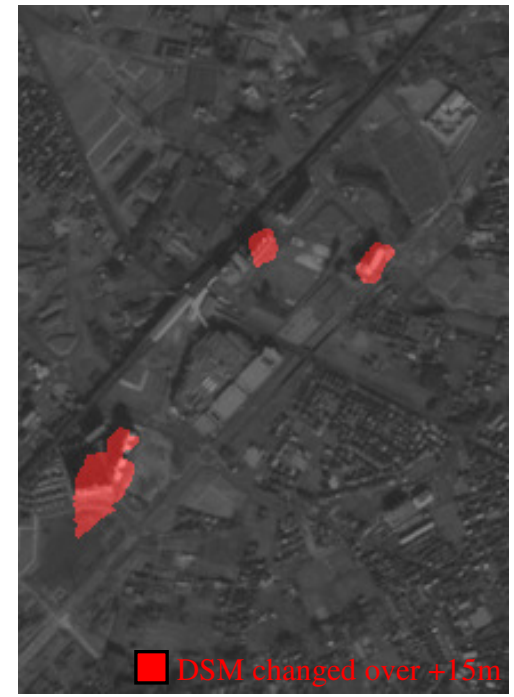

Figure 4: Example of DSM-change image

Left: $1^{\text {st }}$ orthoimage, Center: $2^{\text {nd }}$ orthoimage, Right: DSM-change image overlayed with $2^{\text {nd }}$ orthoimage 

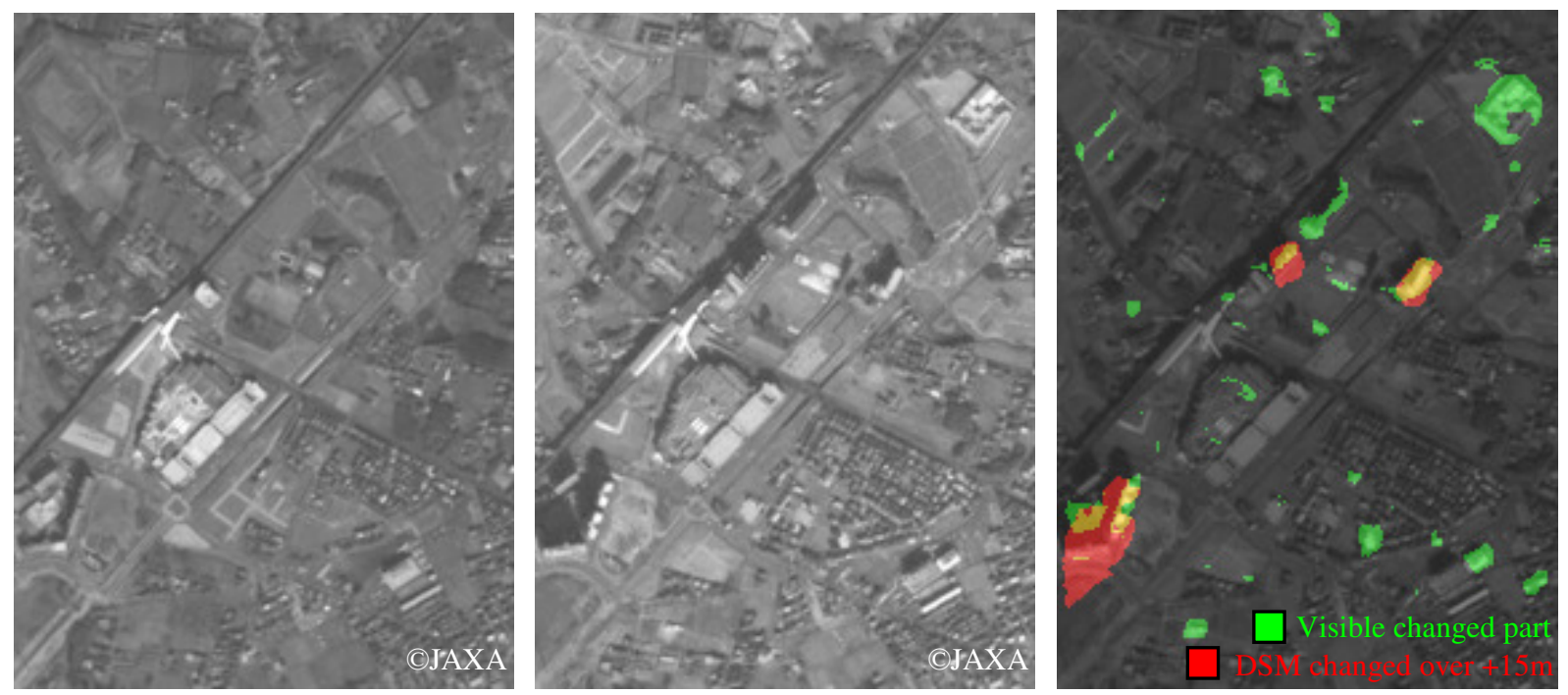

Figure 5: Example of change detected image

Left: $1^{\text {st }}$ orthoimage, Center: $2^{\text {nd }}$ orthoimage, Right: change detected image overlayed with $2^{\text {nd }}$ orthoimage

\subsection{Image integration}

Figure 5 show examples of the change detected image, which is combined with a pixel-change image and a DSM-change image. Here, we assign $\mathrm{G}$ channel to pixel-change, $\mathrm{R}$ channel to DSM-change. Thus, change detected image helps us catching where changes are at a glance.

\subsection{Polygon extraction in elevation changed area}

For further work saving, we developed the method for polygon extraction in elevation changed area. This can help us estimating how many contour lines or building footprints we should update.

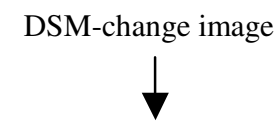

Edge extraction for each elevation changed area

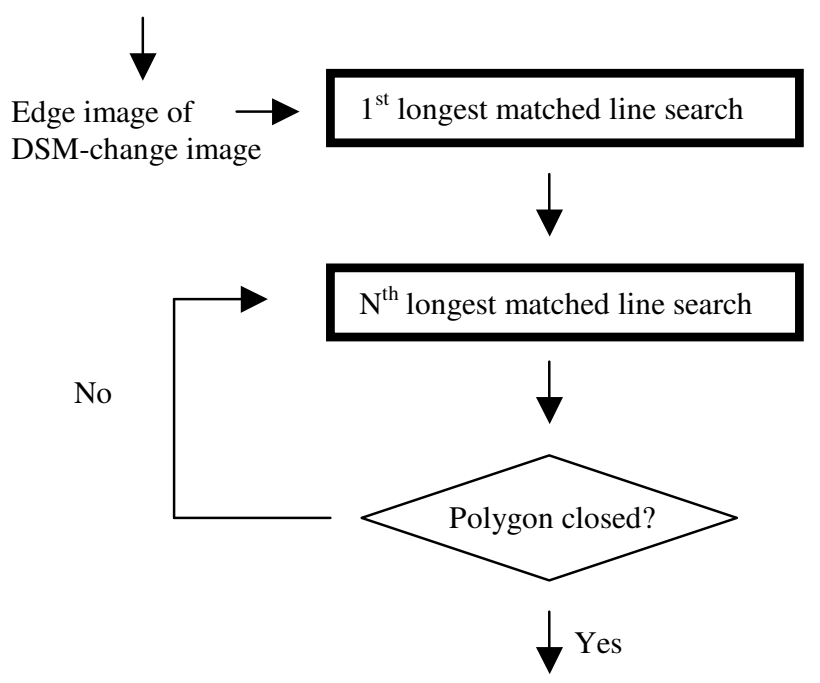

Extracted polygon

Figure 6: Flowchart of polygon extraction method
First, each edge image of elevation changed area in the DSMchange image are extracted. Then this method searches $1^{\text {st }}$ longest matched line with an edge image. In this section, this method makes a line from all points on the bounding rectangle of an edge image to other points on the bounding rectangle. Then it counts the pixel number that the line is on the edge as shown in right part of Figure 7. In this case, there are not so many common pixels on the line. However the left part of Figure 7 shows that this is the case that there are most numerous common pixels on the line. Thus, the longest matched line is selected after the line search with combination of all two points on bounding rectangles.

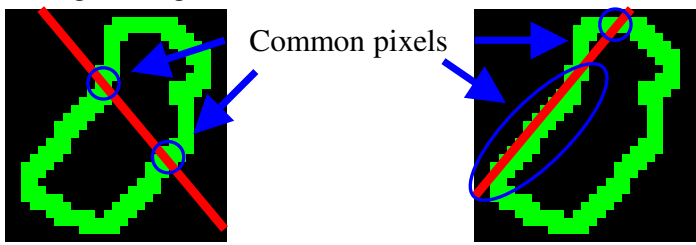

Figure 7: Example of $1^{\text {st }}$ longenst matched line search

Next, $2^{\text {nd }}$ longest matched line is searched. In $1^{\text {st }}$ longest matched line search, this method makes a line from the combination of all two points on the bounding rectangle of edge image. However, from $2^{\text {nd }}$ longest matched line search, it makes a line from the point on every $\mathrm{N}^{\text {th }}$ longest line to all points on the bounding rectangle of edge image shown in Figure 8.

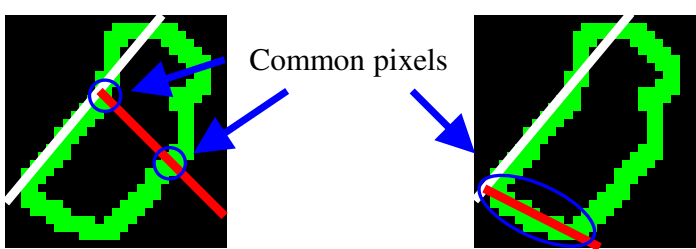

Figure 8: Example of $2^{\text {nd }}$ longenst matched line search

Until the two terminated nodes on the longest lines get same coordinate, this operation repeats. This method can extract line polygons with simple edge images. However this method can't extract line polygons perfectly with edge images with complex shape. We are now improving the algorithm to extract line polygons precisely. 
Table 1: Specification of used images and condition

\begin{tabular}{|l|l|l|}
\hline & $1^{\text {st }}$ images & $2^{\text {nd }}$ images \\
\hline Image area & \multicolumn{2}{|c|}{ Around Tsukuba city, Japan } \\
\hline Observing date & Mar. $1^{\text {st }}, 2007$ & Jan. 22 \\
th, 2010 \\
\hline Ground resolution & \multicolumn{2}{|c|}{$2.5 \mathrm{~m}$} \\
\hline Image-ID(ALPSM) & N058492875 & N212822875 \\
N:Nadir, & F058492820 & F212822820 \\
F:Forward, & B058492930 & B212822930 \\
B:Backword & \multicolumn{2}{|c|}{$2.5 \mathrm{~m}$} \\
\hline $\begin{array}{l}\text { Orthoimage } \\
\text { resolution }\end{array}$ & \multicolumn{2}{|c|}{$5 \mathrm{~m}$} \\
\hline DSM grid size & 12 \\
\hline $\begin{array}{l}\text { Number of GCPs } \\
\text { for orientation with }\end{array}$ & 13 & \\
RPCs & & \\
\hline
\end{tabular}

Table 2: Number of extracted polygons

\begin{tabular}{|l|l|l|l|}
\hline \multicolumn{2}{|c|}{} & \multicolumn{2}{|l|}{ Our method } \\
\cline { 3 - 4 } \multicolumn{2}{|c|}{} & Extracted & Didn't extract \\
\hline $\begin{array}{l}\text { Manual } \\
\text { operation }\end{array}$ & Extracted & 15 & 3 \\
\cline { 2 - 4 } & Didn't extract & 4 & \\
\hline
\end{tabular}

\section{TEST TRIAL AND RESULT}

We have used two sets of ALOS-PRISM triplet images to confirm our method. Table 1 shows the specification of used images and software we used. In addition, we used SAT-PP for generating orthoimages and DSMs.

Figure 9 and Figure 10 (as shown in the next page) show the result of our method. From two DSMs and two orthoimages, change detected image with polygons in elevation changed areas is generated. In change detected images, each RGB channel stands for DSM changed over the $+15 \mathrm{~m}$ part $(\mathrm{R})$, visible changed part $(\mathrm{G})$ and DSM changed under the $-15 \mathrm{~m}$ part (B). This means that yellow part is the both DSM changed over the $+15 \mathrm{~m}$ and visible changed part.

As shown in Figure 9 and Figure 10 we can easily catch where the changed areas are if we see just a change detected image with a visible changes and DSM changes.

We have verificated our method comparing the number of extracted polygons by our method with manual operation in 20 square $\mathrm{km}$ around Tsukuba area. Table 2 shows the number of correct (both our method and manual operation extracted), incorrect (our method extracted while manual operation didn't) and mis-extracted (our method didn't extract while manual operation did).

Our method extracted 19 polygons. However 4 polygons are incorrect. The reason of these all 4 incorrects are due to DSM error. In forests area or paddy field area, there are the cases that there are actually no changes between 2 images, while DSM has an error.

Our method also couldn't extract 3 polygons. These are since each DSM for several buildings are combined with one DSM. Due to this, our method can't extract each separated buildings as shown in Figure 11. In this case there are 2 new buildings, however there is only one extracted polygon. This is correct in change detection, while incorrect in polygon extraction.

\section{CONCLUSION}

In this paper we proposed a method for automatic change detection using pixel changes and DSM changes using ALOS PRISM triplet images. Also we developed an automatic polygon extraction for elevation (DSM) changed part. From the result,
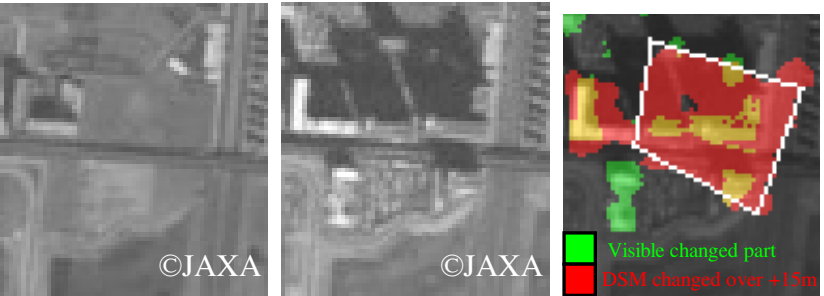

Figure 11: Example of conbined DSM

Left: $1^{\text {st }}$ orthoimage

Cnter: $2^{\text {nd }}$ orthoimage,

Right: change detected image with a polygon overlayed with $2^{\text {nd }}$ orthoimage

we can easily catch where it changes. Our method is not perfect yet so far since there are several mis-extracted and incorrect extractions due to DSM errors. However we can catch the rough change estimation for 1:25000 map data with a glance to change detected images.

We will further improve our method to generate more precise polygons for automatic digitization with new buildings. Also we plan to apply this method to not only ALOS PRISM images but also aerial photos.

\section{REFERENCES}

\section{References from Journals:}

Eisenbeiss H., Baltsavias E. P., Pateraki M., Zhang L., 2004. Potential of IKONOS and QUICKBIRD Imagery for Accurate 3D-Point Positioning, Orthoimage and DSM Generation. International Archives of Photogrammetry and Remote Sensing, 35 (B3), pp. 522-528

Gruen, S.Kocaman, K.Wolff, 2007. "CALIBRATION AND VALIDATION OF EARLY ALOS/PRISM IMAGES" Journal of the Japan Society of Photogrammetry and Remote Sensing, Vol.46, No.1, pp.24-38

\section{References from Other Literature:}

Gruen A., Eisenbeiss H., Hanusch T., Sauerbier M., Wolff K., 2006. Documentation of the reference DSMs of the ALOS testfield Bern/Thun, Switzerland. Report to JAXA, Japan.

A. Sasagawa, 2007. Automatic Change Detection Using Pair of PRISM Triplet Images Proc. The First Joint PI symposium of ALOS Data Nodes for ALOS Science Program in Kyoto, pp.2438

A. Sasagawa, K. Watanabe, S. Nakajima, K.Koido, H.Ohno, H. Fujimura, 2008. Automatic Change Detection Based on Pixelchange and DSM-change, The International Archives of the Photogrammetry, Remote Sensing and Spatial Information Sciences, Beijing, China, Vol. XXXVII, Part B7, pp. 16451650. 

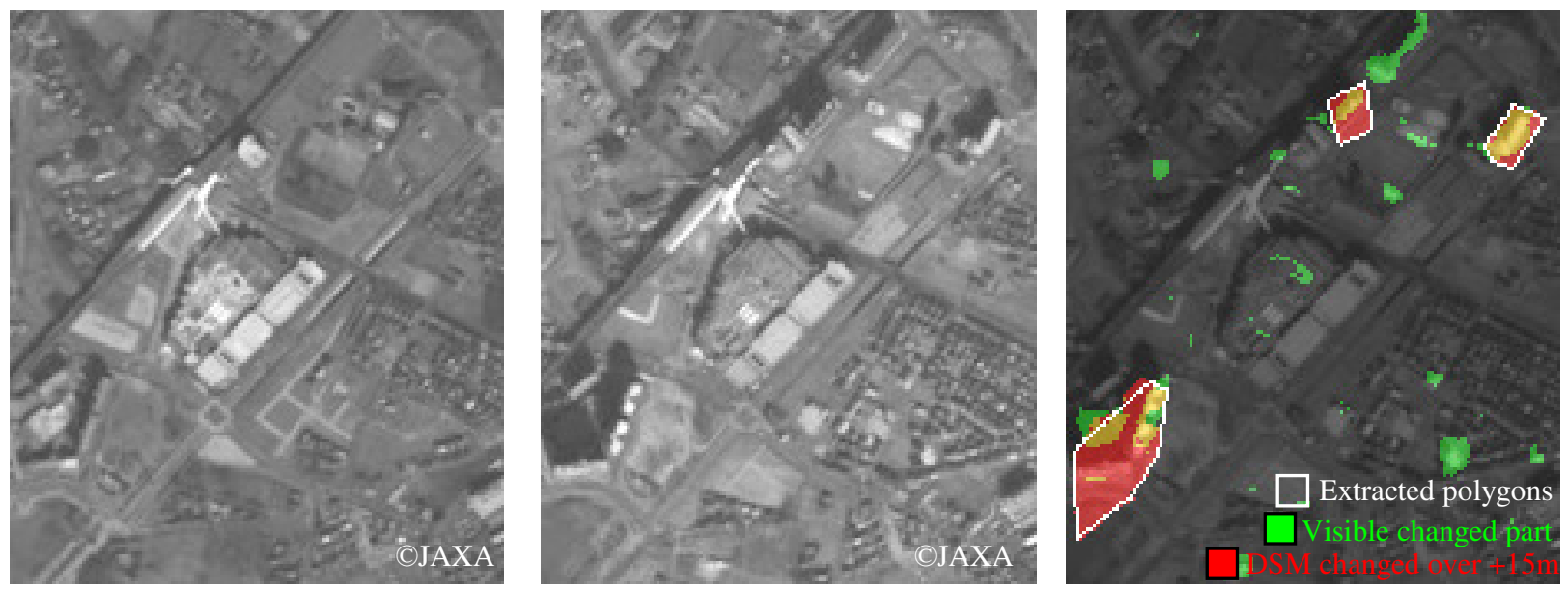

Figure 9: Part of change detected image

Leftt: $1^{\text {st }}$ orthoimage, Center: $2^{\text {nd }}$ orthoimage, Right: change detected image overlayed with $2^{\text {nd }}$ orthoimage and extracted polygons
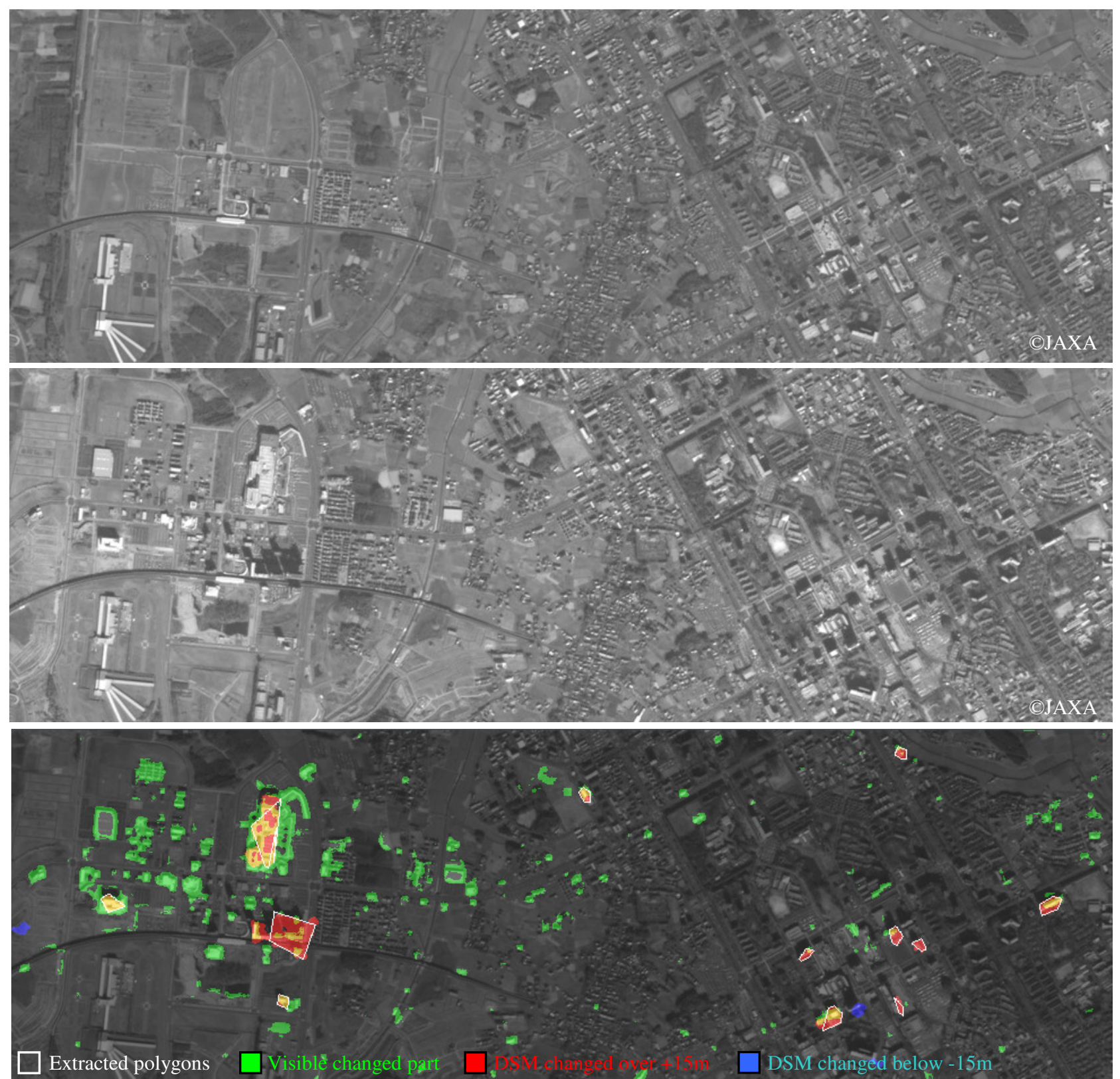

Figure 10: Part of change detected image

Top: $1^{\text {st }}$ orthoimage, Center: $2^{\text {nd }}$ orthoimage, Bottom: change detected image overlayed with $2^{\text {nd }}$ orthoimage and extracted polygons 